\title{
PARTIAL LATTICE PARTICIPATION IN THE SPIN-LATTICE RELAXATION OF POTASSIUM CHROMIUM ALUM
}

\author{
J.A. Overweg, J. Flokstra, H.J.M. ter Brake and G.J. Gerritsma \\ Twente University of Technology \\ Department of Applied Physics \\ P.0. Box 217,7500 AE ENSCHEDE \\ The Netherlands
}

We developed a SQUID-based frequency sweeping system for a.c. susceptibility measurements. Using this instrument we found that in Potassium Chromium Alum only a part of the lattice system is involved in the spin-lattice relaxation process. This partial lattice participation amounts $60-75 \%$ of the total lattice specific heat.

\section{PARTIAL LATTICE PARTICIPATION}

In the direct and Orbach spin-lattice relaxation processes only narrow bands of phonons are on speaking terms with the spins. The problem we are investigating is the following: is the surplus energy in the resonant bands transferred directly to the environment of the sample or also to other phonon modes. We can obtain information about this by measuring the frequency dependence of the susceptibility of a sample both when it is thermally isolated (vacuum) from the surrounding helium bath and when it is in good thermal contact with its environment [1]. The specific heat $C_{L}{ }^{*}$ of the part of the lattice which is involved in the relaxation obeys the relation

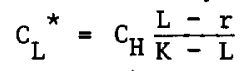

where $C_{H}$ is the spin specific heat, $K$ and $L$ are the isothermal (low frequency) limits of the susceptibility (relative to $\chi_{T}(H=0)$ ) in case of good thermal contact and in vacuum respectively while $r$ is the adiabatic (high frequency) limit of $x$.

In previous experiments on Cerium Magnesium Nitrate, where the spin-lattice relaxation is governed by the Orbach process, we found, that the thus obtained lattice specific heat is exactly equal to the calorimetrically determined value [2], indicating that all the lattice oscillators participate in the spin-lattice relaxation. Obviously, in this substance the interaction between high frequency phonons and the other (thermal) phonons is sufficiently good to establish thermal equilibrium within the lattice. Preliminary experiments on Potassium Chromium alum, where the direct process is dominant, showed, that there the specific heat of the part of the phonon system which is involved in the relaxation process is smaller than the calorimetric specific heat [1] (partial lattice participation, see fig. 3). Because these measurements were done with a conventional inductance bridge the low frequency limits of $x$ could then only be obtained by extrapolation so there was still some doubt about the correctness of these results.

\section{THE NEW SQUID-SUSCEPTOMETER}

In order to be able to do accurate ACsusceptibility measurements at very low frequencies we have developed a susceptometer in which the off-balance signal of a primarysecondary coil set is detected with a SQUID (fig. 1.). The sensitivity of this instrument is basically independent of the frequency of the input signal. In our first prototype [3] we used a lock-in amplifier (lowest frequency $1.5 \mathrm{~Hz}$ ) to analyse the output signal. The low frequency capabilities of the instrument have now been considerably improved by the use of an on-line minicomputer to process the output voltage of the SQUID. It calculates the magnitudes of the in phase ( $\chi^{\prime}$, dispersion) and quadrature ( $\chi^{\prime \prime}$, absorption) components of the signal, it corrects for linear drifts of the output signal and also for jumps of the SQUID to another lockpoint. We can now easily go down to frequencies as low as $0.01 \mathrm{~Hz}$.

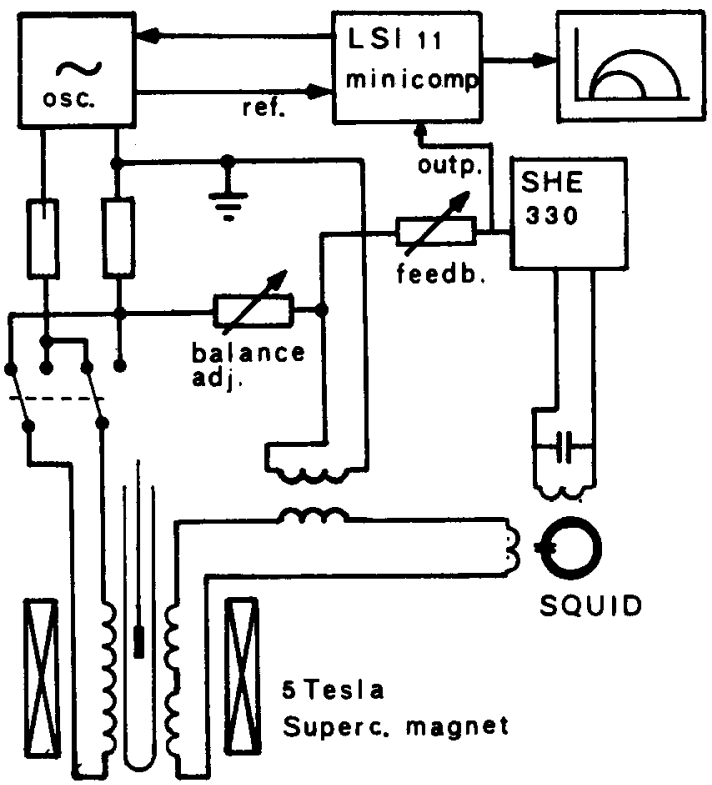

fig. 1. Diagram of the susceptometer. 
Apart from the high sensitivity at low frequencies the instrument offers several other advantages over conventional mutual inductance systems. Because of the frequency-independent response of the SQUID we are able to sweep the frequency of the AC field. The frequency setting of the oscillator is controlled by the computer and the system fully automatically produces plots of $X^{\prime \prime}$ vs. $X^{\prime}$ or of $X^{\prime}$ and $X^{\prime \prime}$ vs frequency. A typical example of such a frequency sweep is shown in fig. 2. It takes only a few minutes to produce such a plot. Further, as the experiments are carried out at constant field, we can investigate samples in vacuum or in helium gas at saturation pressure. In the latter case a thin helium film is adsorbed at the surface of the sample, which provides a much better thermal stabilization than a pool of liquid helium (note the diffence of the two upper curves in fig. 2). With conventional inductance bridges, where usually the magnetic field is swept instead of the frequency, it is very difficult to work under such experimental conditions, because of the large magnetocaloric effects, that occur when the external field is changed.

\section{RECENT EXPERIMENTAL RESULTS ON $\mathrm{K}$ Cr ALUM}

With our new SQUID-system we were able to measure the low frequency limits of $x(\omega)$ far more accurately. The now found partial lattice participation basically confirms our earlier results. We measured the specific heat of the part of the lattice which is active in the relaxation for a cylindrical sample of $\mathrm{K} \mathrm{Cr}$ alum (03mm, length $10 \mathrm{~mm}$ ) in the temperature range from $2 \mathrm{~K}$ to $4.2 \mathrm{~K}$, at a constant field of $0.5 \mathrm{~T}$. As shown in fig. 3. about $60 \%$ of of the lattice is involved in the relaxation between $2 \mathrm{~K}$ and $3.3 \mathrm{~K}$, this fraction increases to $75 \%$ at $4.2 \mathrm{~K}$. We found no significant field dependence of this partial lattice participation between 0.15 and 1.5 Tesla. We did not observe equilibrium within the lattice on decreasing the frequency down to

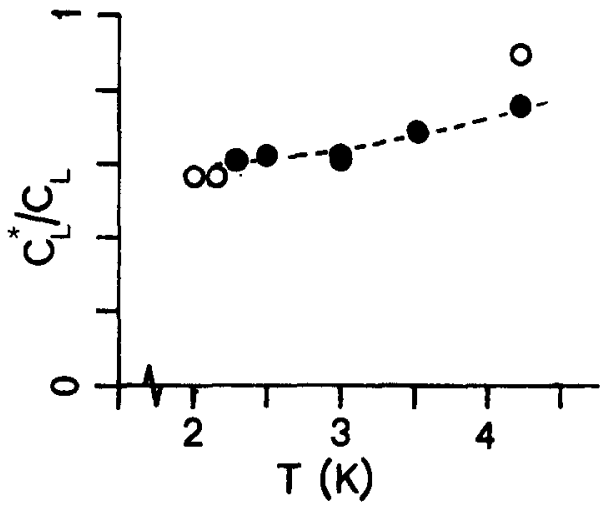

fig. 3. Partial lattice participation in $\mathrm{K} \mathrm{Cr}$ alum.

- old measurements, - recent results.

$0.01 \mathrm{~Hz}$, so we may conclude, that the time constant of this lattice relaxation process is at least $100 \mathrm{~s}$.

\section{ACKNOWLEDGEMENTS}

We wish to thank W.J. van Weezep for the development of the software for our susceptometer

\section{REFERENCES :}

[1] Flokstra, J., Gerritsma, G.J. and Van der Marel, L.C., Spin-lattice relaxation in Potassium Chromium Alum, Physica 94B (1978) 53.

[2] Overweg, J.A., Flokstra, J, and Gerritsma, G.J., Spin-lattice relaxation in Cerium Magnesium Nitrate. No partial lattice participation observed. Proc. ICM, Munich 1979,739.

[3] Overweg, J.A., Flokstra, J, and Gerritsma, G.J., A SQUID-based frequency sweeping system for AC susceptibility measurements. Proc. Ismar Ampère, Delft 1980.

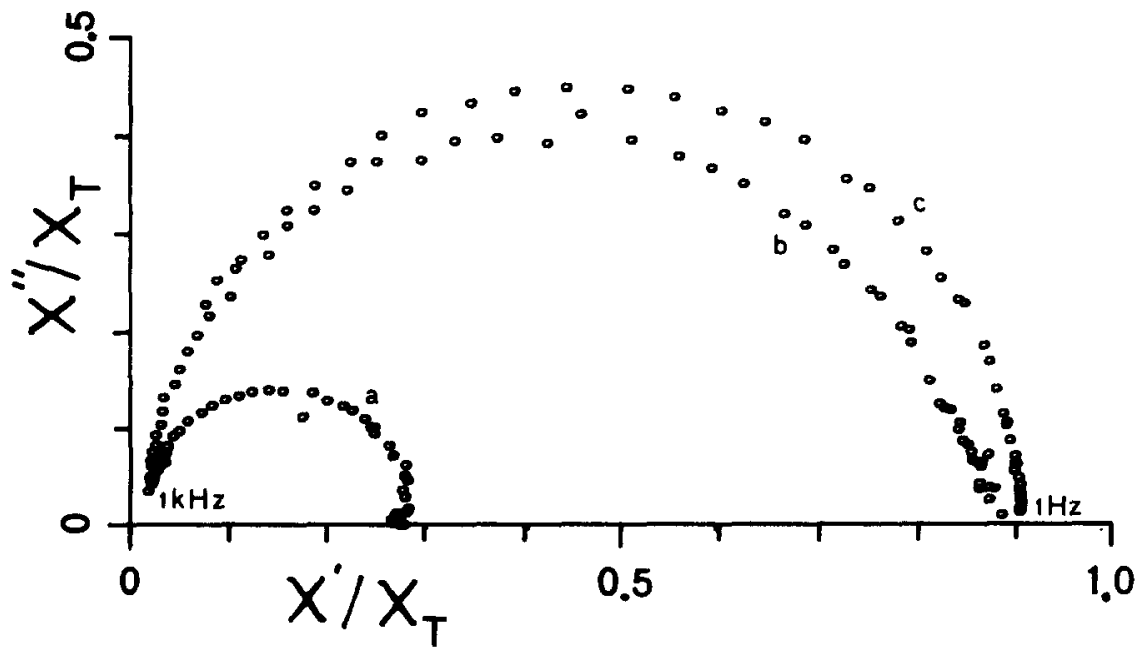

Fig. 2. Examp1e of frequency sweep.

$\mathrm{K} . \mathrm{Cr}$ alum, 4.2K, $0.75 \mathrm{~T}$

a) sample in vacuum;

b) in liquid helium;

c) in helium gas at saturation pressure. 